\title{
REFUERZO DE ELEMENTOS ESTRUCTURALES DE HORMIGON ARMADO MEDIANTE ENCOLADO DE BANDAS DE ACERO CON ADHESIVOS EPOXIDICOS
}

\author{
(STRENGTHENING OF STRUCTURAL ELEMENTS OF REINFORCED CONCRETE BY MEANS OF \\ GLUED STEEL PLATES WITH EPOXY ADHESIVES)
}

Manuel Fernández Cánovas

Dr. Ingeniero de Construcción

IETCC/CSIC

$679-12$

\section{RESUMEN}

El refuerzo de elementos estructurales de hormigón armado, sometidos a flexión, mediante el encolado con un adhesivo epoxi de la armadura precisa en forma de bandas de acero, es un sistema cada día más utilizado y que, sin embargo, en muchos casos, por un mal conocimiento de su técnica, el refuerzo no funciona como debiera o deja de ser eficaz al cabo de cierto tiempo, provocando incluso el hundimiento de la estructura.

En este artículo el autor resume una parte del trabajo llevado a cabo en el Instituto Eduardo Torroja del Consejo Superior de Investigaciones Científicas, y en el que se estudian los diferentes parámetros que pueden influir en la eficacia de este tipo de refuerzos.

\section{SUMMARY}

The strengthening of structural elements of reinforced concrete submitted to bending, by means of sticking with an epoxyadhesive of the precise structure in shape of, steel bands is a more and more used system. But, however, in many cases the strengthening to not runs properly or is no more effective after a while due to a bad knowledge of its technig, causing the structure to sink.

In this report the author resumes part of the work carried out by the Eduardo Torroja Institute from the Higher Scientific Research Council, and studies the different parameters that could influence on the efficacity of this kind of strengthening.

\section{INTRODUCCION}

Con relativa frecuencia se presenta el problema de tener que reforzar un edificio existente o elementos de la estructura del mismo como consecuencia de un cambio de uso, de disminución de resistencia de los elementos debida a acciones accidentales, errores en el diseño o en la ejecución de la estructura, etcétera.

En la solución de este problema es posible emplear diferentes técnicas de refuerzo, pero, quizá una de las que está más en boga, sea la de introducir la armadura suplementaria que necesita el elemento trabajando a flexión, en forma de una banda de acero, de la sección precisa encolada, a las superficies del hormigón y en las zonas convenientes, por medio de un adhesivo epoxi.

Este tipo de refuerzos posee ventajas muy notables sobre los clásicos y que pueden resumirse en los siguientes: rapidez de ejecución, bajo costo, no precisar instalaciones auxiliares importantes, no trabajar con materiales mojados o húmedos, etc.; sin embargo, frente a estas ventajas hay que contar con el inconveniente de su sensibilidad a los fallos que puedan cometerse en su proyecto, realización y, especialmente, en la elección del adhesivo epoxi. El desconocimiento en la forma de trabajar el refuerzo en su conjunto con el hormigón, en el dimensionamiento de la sección de acero y longitud de la banda, deficiencias en la preparación de las superficies a unir, en el espesor de la capa de adhesivo, en la elección del espesor de la banda de acero, etc., pueden tener efectos muy desfavorables sobre la eficacia del refuerzo.

Si el conocimiento de la forma de trabajar este tipo de refuerzos es fundamental, no lo es menos el de las características de las formulaciones epoxídicas utilizadas. Existe una tendencia generalizada a utilizar formulaciones epoxi universales que sirven lo mismo para unir dos hormigones, que para revestir un pavimento o para hacer un refuerzo estructural y nada más lejos de la realidad; una formulación epoxi para este tipo de refuerzos debe poseer unas características acordes con la forma en que ha de trabajar dentro del refuerzo y que, en general, son muy diferentes a las exigidas en otras 
aplicaciones; no hay que olvidar que un adhesivo epoxi actúa en este caso como transmisor de acciones del hormigón al acero y que cualquier ineptitud se traducirá en que, a corto o largo plazo, el acero deje de cumplir con la misión de refuerzo que se le ha encomendado.

En 1973 iniciamos, en el Instituto Eduardo Torroja, los primeros ensayos a escala reducida sobre vigas reforzadas con bandas de acero encoladas al hormigón con resinas epoxi, dándonos cuenta de la importancia del tema y de las lagunas que presentaba. Tratando de estudiar más a fondo esta técnica solicitamos una subvención a la Comisión Asesora de Investigación Científica y Técnica del Ministerio de Educación y Ciencia para llevar a efecto un programa de investigación completo sobre este tipo de refuerzos. La CAICYT consideró de interés el tema presentado y gracias a su ayuda hemos podido llevar a cabo este proyecto, algunos de cuyos resultados presentamos en este artículo, muy resumidos por razones de espacio, aunque próximamente podrán contemplarse en su totalidad en una monografía que publicará el Instituto.

\section{PARAMETROS ESTUDIADOS}

El trabajo realizado ha constado de tres partes fundamentales: una primera en la que se han estudiado las formulaciones epoxi empleadas en los ensayos y en el que se han efectuado los ensayos oportunos para determinar las características de las mismas, una segunda en la que se ha estudiado el comportamiento de los refuerzos frente a acciones diferentes y una tercera en la que se ha llevado a cabo un estudio teórico completo de la forma de trabajar estos refuerzos.

El trabajo termina con una serie de recomendaciones para conseguir la mayor eficacia de este tipo de refuerzos.

En este artículo nos hemos centrado exclusivamente en la segunda parte del estudio, es decir, en su fase experimental, dando unas conclusiones finales así como unas recomendaciones de empleo.

\section{FORMULACIONES EPOXI EMPLEADAS}

En los trabajos realizados hemos utilizado tres tipos de adhesivos epoxídicos recomendados por casas de rango internacional para este tipo de refuerzos y que en lo sucesivo designaremos como Adhesivos tipo «A», «B» $y$ «C». Estos adhesivos entre otras características poseen las que resumimos en el cuadro n. ${ }^{\circ} 1$.

\section{Cuadro 1}

\begin{tabular}{|c|c|c|c|}
\hline Adhesivo tipo & A & B & $\mathrm{C}$ \\
\hline $\begin{array}{l}\text { Resist. compresión } \\
\text { a } 7 \text { días y } 23^{\circ} \mathrm{C} \\
\left(\mathrm{MN} / \mathrm{m}^{2}\right)\end{array}$ & 82,3 & 93,0 & 60,9 \\
\hline $\begin{array}{l}\text { Resist. flexotracción } \\
\text { a } 7 \text { días y } 23{ }^{\circ} \mathrm{C} \\
\left(\mathrm{MN} / \mathrm{m}^{2}\right)\end{array}$ & 28,9 & 35,0 & 36,5 \\
\hline $\begin{array}{l}\text { Resist. cizallamien- } \\
\text { to acero-hormigón a } \\
7 \text { dias y } 23{ }^{\circ} \mathrm{C}(\mathrm{MN} / \\
\left.\mathrm{m}^{2}\right)\end{array}$ & 6,6 & 6,5 & 6,0 \\
\hline $\begin{array}{l}\text { Módulo elasticidad } \\
\text { a compresión }(\mathrm{MN} / \\
\left.\mathrm{m}^{2}\right)\end{array}$ & $15 \times 10^{3}$ & $17 \times 10^{3}$ & $7,5 \times 10^{3}$ \\
\hline $\begin{array}{l}\text { Módulo de rigidez } \\
\text { transversal }\left(\mathrm{MN} / \mathrm{m}^{2}\right)\end{array}$ & & $6,7 \times 10^{3}$ & \\
\hline Pot life $20^{\circ} \mathrm{C}$ & $\begin{array}{l}45 \mathrm{~min} \\
(5 \mathrm{~kg})\end{array}$ & $\begin{array}{l}120 \mathrm{~min} \\
(5 \mathrm{~kg})\end{array}$ & $\begin{array}{l}40 \mathrm{~min} \\
(2 \mathrm{~kg})\end{array}$ \\
\hline $\begin{array}{l}\text { Relación compo- } \\
\text { nentes (en peso): } \\
\text { Resina cargada/en- } \\
\text { durecedor cargado }\end{array}$ & 1,70 & 2,85 & 3,1 \\
\hline
\end{tabular}

\section{PLAN DE ENSAYOS}

El plan de ensayos de este trabajo ha supuesto la rotura de 109 vigas de $3,35 \mathrm{~m}$ de longitud, $0,25 \mathrm{~m}$ de ancho y $0,20 \mathrm{~m}$ de canto, fabricadas con hormigón de resistencia característica $\mathrm{f}_{\mathrm{ck}}=20 \mathrm{MN} / \mathrm{m}^{2}$ y armadas con $4 \varnothing 8$ de acero de $\mathrm{f}_{\mathrm{yk}}=540 \mathrm{MN} / \mathrm{m}^{2}$, dos en la zona comprimida $\mathrm{y}$ dos en la tendida (Fig. 1).
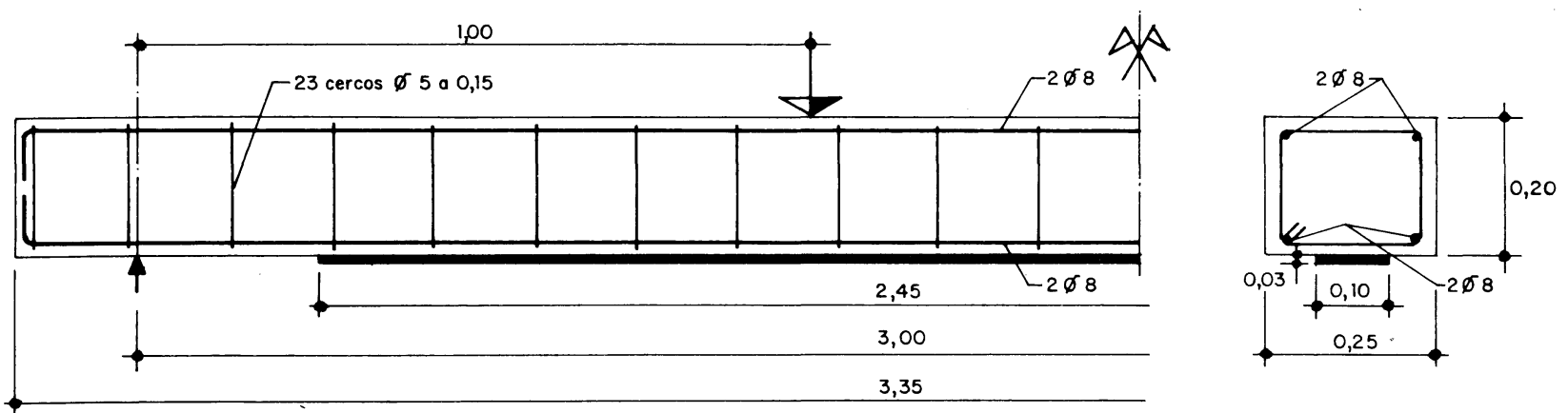

Fig. 1.-Viga reforzada tipo. 

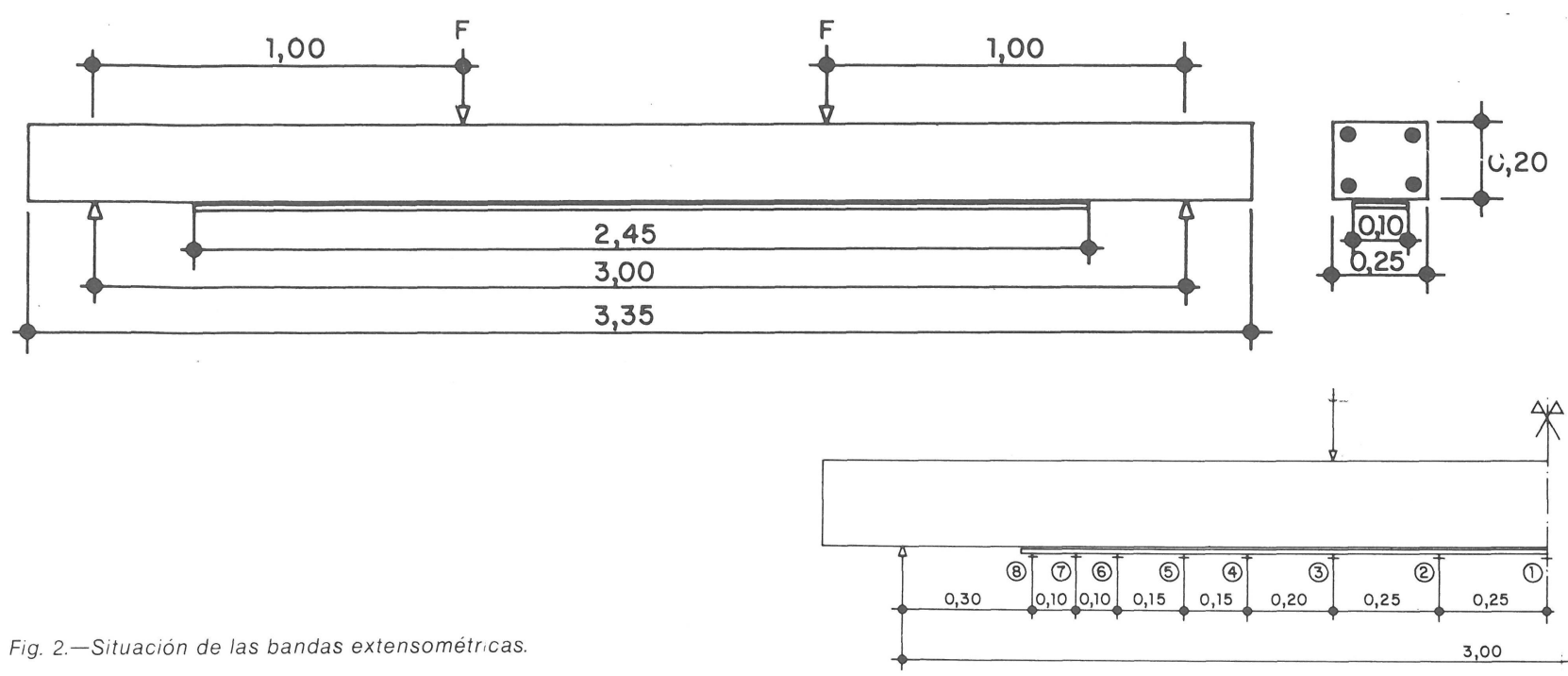

Las vigas así constituidas, y sobre las que se aplicaría el refuerzo, mostraban un momento de rotura de cálculo de 9,6 mKN. Sometidas a ensayo de flexión presentaban fisuras de $0,05 \mathrm{~mm}$ y una flecha en el centro de $1,47 \mathrm{~mm}$ para un momento de $3 \mathrm{mKN}$ y fisuras de $0,8 \mathrm{~mm}$ y flecha de 22,6 $\mathrm{mm}$ para un momento de $9 \mathrm{mKN}$. Para este momento el hormigón estaba trabajando en la zona comprimida a $19,3 \mathrm{MN} / \mathrm{m}^{2}$ y el acero de armado había superado el límite elástico. La rotura se produjo para un momento de $12 \mathrm{mKN}$.

Estas vigas tipo se han reforzado con bandas de acero A-37, encoladas con adhesivos epoxi tipos A, B y C, a fin de estudiar la influencia que en la eficacia del refuerzo tienen los siguientes parámetros:

- preparación de las superficies a unir en hormigón y acero,

- resistencia característica del hormigón,

- tipo de adhesivo,

- espesor de la capa de adhesivo,

- espesor de la banda de refuerzo,
- fatiga,

- cargas mantenidas o fluencia.

Todos los ensayos se han realizado a flexión sobre una luz libre de 3,00 m y con dos cargas iguales crecientes aplicadas a un tercio de la luz, midiéndose en ellos las flechas de las vigas, las deformaciones en las fibras comprimidas y tendidas a la altura de las barras de armado, así como las tensiones que aparecen para cada escalón de carga en distintos puntos de las placas de refuerzo por medio de bandas extensométricas cuya situación puede verse en la figura 2.

Todas las vigas reforzadas se han comparado con otra, tipo reforzada, con una banda de acero de $2,45 \times 0,10 \times 0,003 \mathrm{~m}$ encolada con un adhesivo epoxi tipo $\mathrm{A}$ y en la que la preparación de las superficies, tanto del hormigón como del acero, se había realizado con chorro de arena. En esta viga se había producido la rotura para un momento de $32 \mathrm{mKN}$, superándose ampliamente el límite elástico de acero, de armado y de refuerzo en la zona central de la viga y ocurriendo la rotura por plastificación del hormigón en la

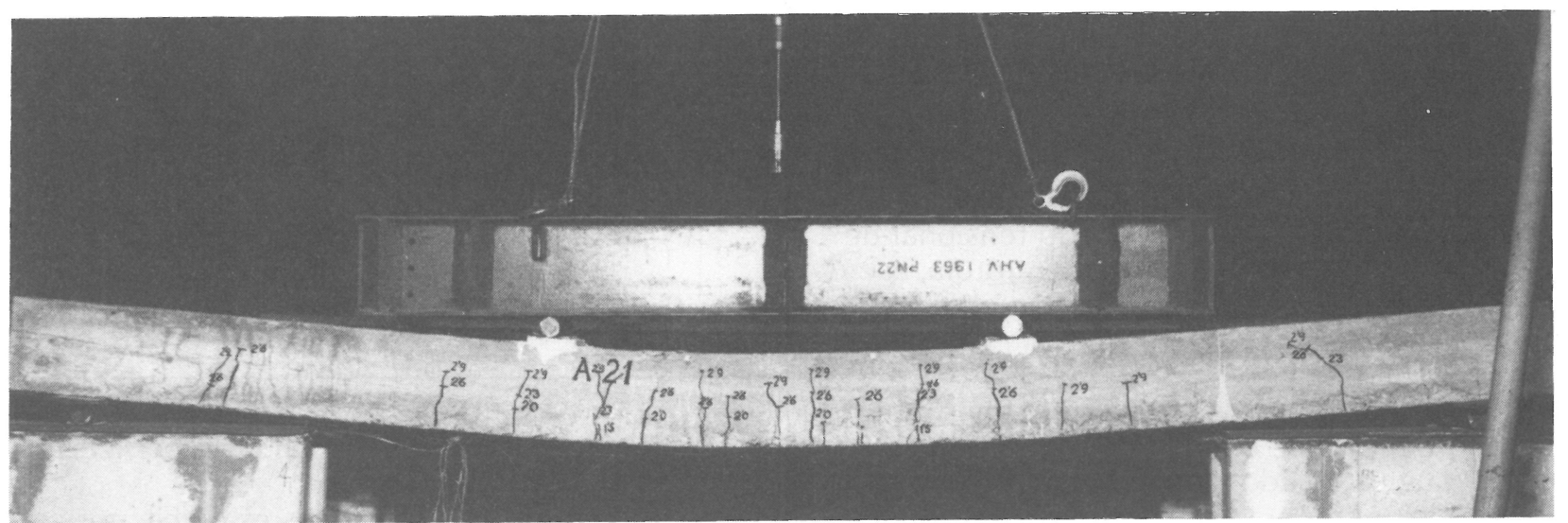




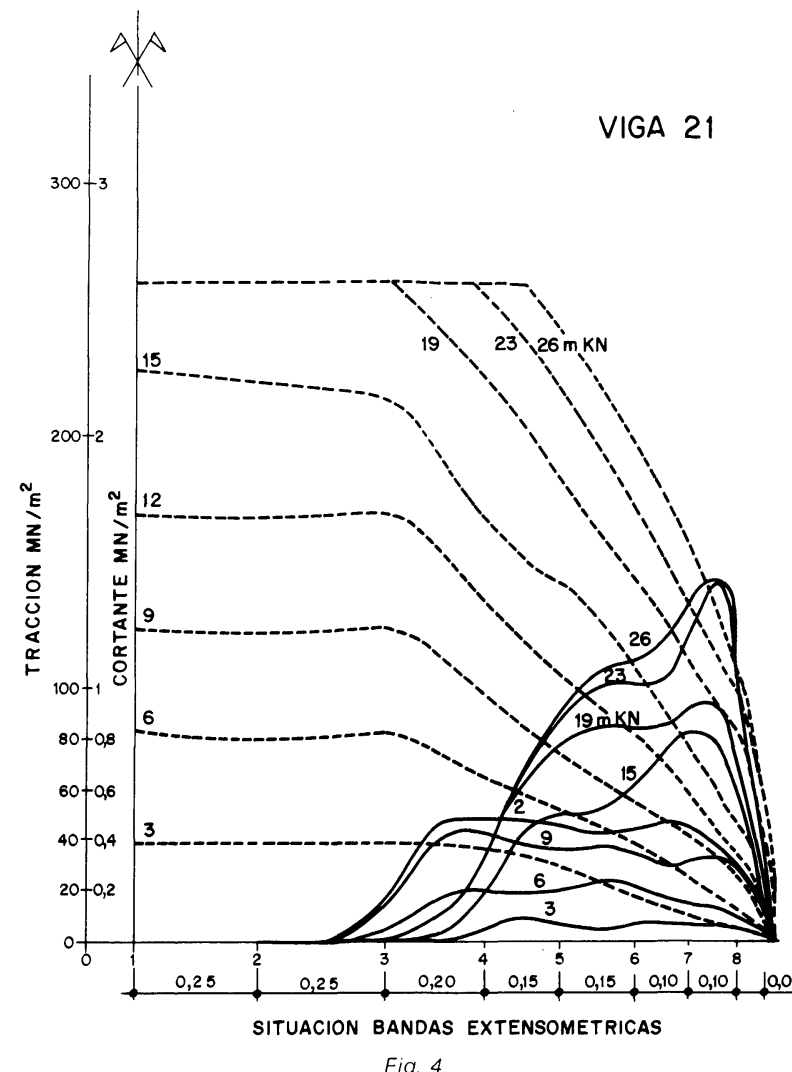

Fig. 4

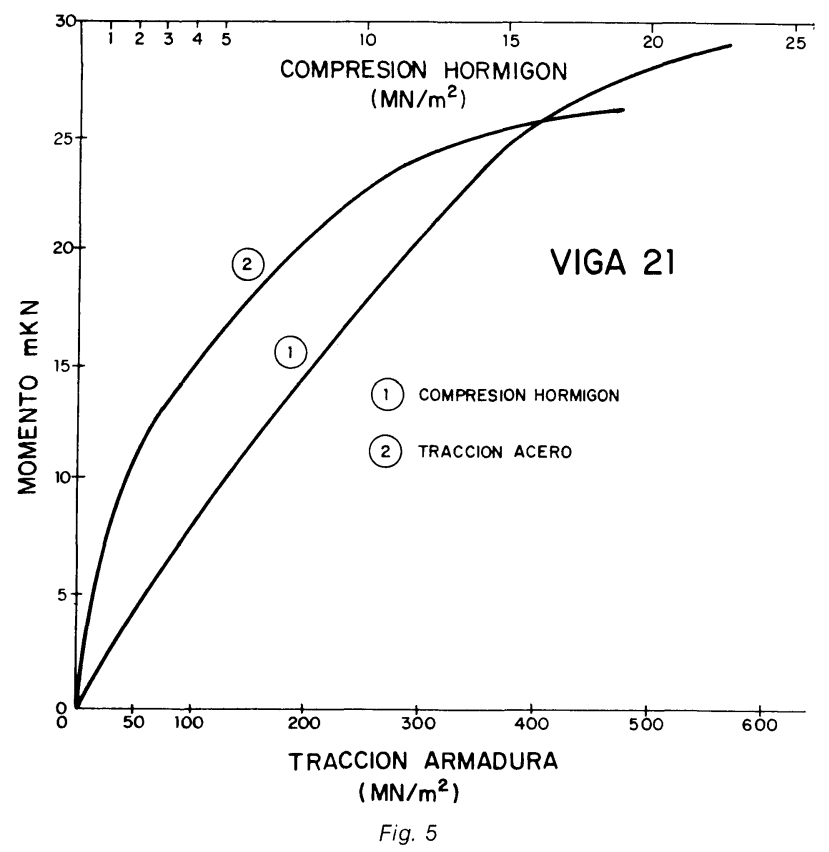

zona comprimida (Fig. 3). El estado tensional de la banda de refuerzo y de la viga en rotura, así como las flechas producidas, pueden observarse en las figuras 4,5 y 6 .

El haber utilizado en los ensayos el adhesivo $\mathrm{A}$ como tipo o patrón, era debido a que este tipo estaba muy estudiado y experimentado a escala laboratorio, y en obra por la casa Ciba-Geygy de Basilea (Suiza).

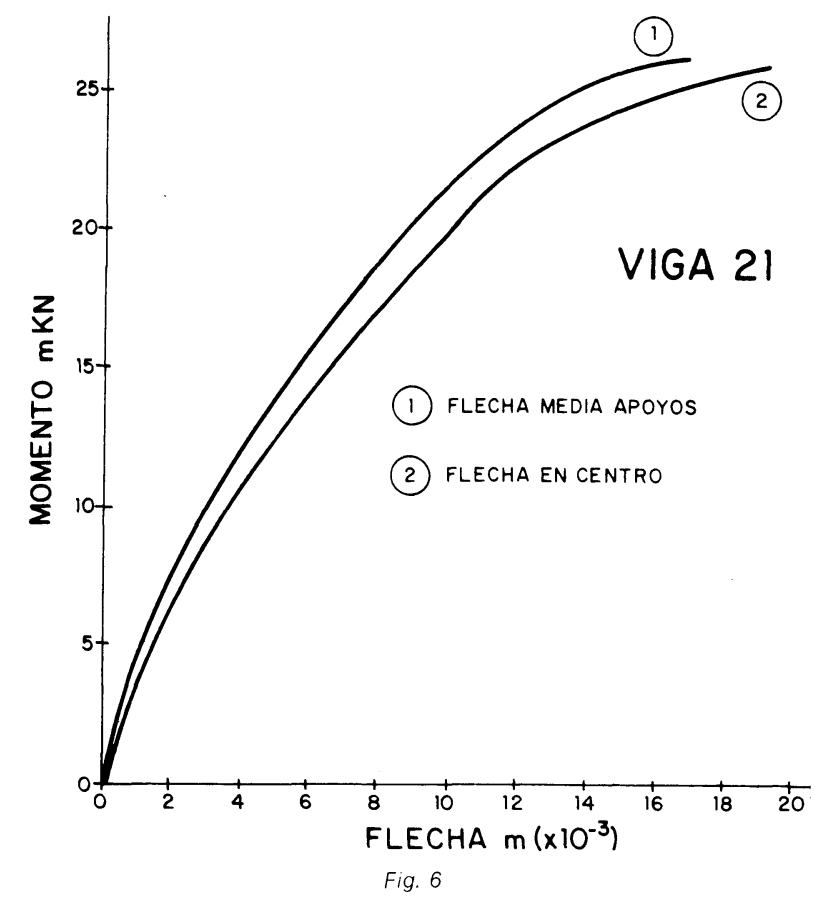

\subsection{Influencia de la resistencia característica del} hormigón

Para estudiar la influencia de la resistencia en la eficacia del refuerzo, así como el valor de ésta por debajo del cual el refuerzo deja de ser eficaz, se han ensayado vigas reforzadas con bandas de acero iguales a la empleada en la viga tipo de comparación y en las que el hormigón tenía resistencias características de $25,0,20,0,17,5$ y $15,0 \mathrm{MN} / \mathrm{m}^{2}$.

Los resultados obtenidos en los ensayos figuran en el Cuadro 2, en el que se indican los momentos de servicio y último de rotura de cálculo, así como el momento último de agotamiento en el ensayo.

\section{Cuadro 2}

\begin{tabular}{|c|c|c|c|c|}
\hline $\begin{array}{c}\text { Viga } \\
\text { n. }\end{array}$ & $\begin{array}{c}\mathrm{f}_{\mathrm{ck}} \\
\mathrm{MN} / \mathrm{m}^{2}\end{array}$ & $\begin{array}{c}\text { Momento de } \\
\text { servicio } \mathrm{M} \mathrm{mKN}\end{array}$ & $\begin{array}{c}\text { Momento } \\
\text { último } \mathrm{M}_{\mathrm{u}} \mathrm{mKN}\end{array}$ & $\begin{array}{c}\text { Momento de } \\
\text { agotamiento mKN }\end{array}$ \\
\hline 43 & & 15,2 & 31,4 & 31,0 \\
\hline 44 & 25,0 & 15,2 & 31,4 & 32,0 \\
\hline 21 & & 14,8 & 29,7 & 31,1 \\
\hline 29 & 20,0 & 14,8 & 29,7 & 32,0 \\
\hline 55 & & 14,7 & 28,9 & 28,5 \\
\hline 59 & 17,5 & 14,7 & 28,9 & 29,5 \\
\hline 45 & & 14,5 & 28,4 & 21,2 \\
\hline 46 & 15,0 & 14,5 & 28,4 & 19,0 \\
\hline
\end{tabular}

\subsection{Influencia de la preparación de superficies}

Dado que en muchas ocasiones es difícil, por razones de uso de la estructura, preparar las 
Cuadro 3

\begin{tabular}{|c|c|c|c|c|c|}
\hline \multirow{2}{*}{$\begin{array}{c}\text { Viga } \\
\text { n. }\end{array}$} & \multicolumn{2}{|c|}{ Tratamiento } & \multirow{2}{*}{$\begin{array}{l}\text { Momento de } \\
\text { agotamiento } \\
\mathrm{mKN}\end{array}$} & \multirow{2}{*}{$\begin{array}{l}\text { Momento de } \\
\text { agotamiento } \\
\text { mKN (medio) }\end{array}$} & \multirow{2}{*}{$\begin{array}{l}\% \text { variación } \\
\text { respecto a } \\
\text { la media }\end{array}$} \\
\hline & Hormigón & Acero & & & \\
\hline $\begin{array}{l}3 \\
5\end{array}$ & Chorro de arena & Chorro de arena & $\begin{array}{l}29,6 \\
29,1\end{array}$ & 29,4 & 0,0 \\
\hline $\begin{array}{l}4 \\
6\end{array}$ & Chorro de arena & $\begin{array}{l}\text { Chorro de arena y } \\
\text { pintado }\end{array}$ & $\begin{array}{l}26,0 \\
26,0\end{array}$ & 26,0 & $-11,5$ \\
\hline $\begin{array}{l}7 \\
8\end{array}$ & Escarificado & Chorro de arena & $\begin{array}{l}28,1 \\
29,0\end{array}$ & 28,6 & $-2,7$ \\
\hline $\begin{array}{r}9 \\
10\end{array}$ & Escarificado & Esmerilado & $\begin{array}{l}28,5 \\
29,0\end{array}$ & 28,7 & $-2,4$ \\
\hline
\end{tabular}

superficies del hormigón y del acero mediante chorreado con arena, empleándose el escarificado del hormigón y el esmerilado del acero de la banda, es por lo que hemos realizado ensayos sobre vigas tipo en las que se habían tenido en cuenta estos tratamientos. Los tratamientos estudiados han sido: chorro de arena sobre hormigón y acero, chorro de arena sobre hormigón y acero, pero con tratamiento posterior de la superficie de éste con una pintura epoxi que se lijaría ligeramente antes de proceder a la adhesión, escarificado del hormigón y chorreado de la banda con arena y, por último, escarificado del hormigón y esmerilado de la superficie de la banda de acero.

Los ensayos realizados y los resultados obtenidos se recogen en el Cuadro 3. En él aparecen los porcentajes de variación de los resultados de los diferentes tratamientos con respecto al de chorreado con arena de hormigón y acero.

\subsection{Influencia del tipo de adhesivo epoxi utilizado}

Para observar la influencia que los diferentes adhesivos empleados en nuestros ensayos tienen, y que hemos designado como adhesivos A, B y C, en la eficacia de los refuerzos, se han ensayado vigas iguales a la tipo de comparación y en las que se han empleado espesores mínimos de cada uno de los adhesivos a estudiar. Todas las vigas ensayadas habían sido tratadas superficialmente mediante chorro de arena tanto en el hormigón como en el acero.

Los resultados resumidos de los ensayos están recogidos en el Cuadro 4.

\subsection{Influencia del espesor de la capa de adhesivo}

En los estudios teóricos, llevados a cabo por Bresson y otros investigadores, se observa que el aumento en el espesor de la capa de adhesivo empleado en el encolado influye negativamente en la eficacia del refuerzo. Por otra parte, a fin de comprobar este extremo, frecuente en obras
Cuadro 4

\begin{tabular}{|c|c|c|c|c|c|}
\hline $\begin{array}{c}\text { Viga } \\
\mathbf{n .}^{\circ}\end{array}$ & $\begin{array}{c}\text { Tipo de } \\
\text { adhesivo }\end{array}$ & $\begin{array}{c}\text { Momento } \\
\text { de } \\
\text { servicio } \\
\mathbf{m K N}\end{array}$ & $\begin{array}{c}\text { Momento } \\
\text { último } \\
\mathbf{m}_{\mathrm{u}} \mathbf{m K N}\end{array}$ & $\begin{array}{c}\text { Momento } \\
\text { de agota- } \\
\text { miento } \\
\text { mKN }\end{array}$ & $\begin{array}{c}\text { \% de } \\
\text { variación } \\
\text { media del } \\
\text { adhesivo A }\end{array}$ \\
\hline 21 & $\mathrm{~A}$ & 14,8 & 29,7 & 31,1 & 0,0 \\
29 & & 14,8 & 29,7 & 32,0 & \\
19 & $\mathrm{~B}$ & 14,8 & 29,7 & 31,5 & $+0,3$ \\
20 & & 14,8 & 29,7 & 31,8 & \\
25 & $\mathrm{C}$ & 14,8 & 29,7 & 30,0 & $-4,6$ \\
26 & & 14,8 & 29,7 & 30,2 & \\
\hline
\end{tabular}

en las que por la existencia de cejas entre tablas de encofrados o falta de planeidad en las superficies de hormigón hay que emplear espesores de adhesivo por encima de los mínimos recomendados, se han realizado ensayos sobre vigas tipo en las que se han empleado adhesivo tipo A y chorreado con arena del hormigón y acero.

Los espesores de la capa de adhesivo han sido $0,7,1,0,2,0,3,0$ y $5,0 \mathrm{~mm}$, y los resultados obtenidos en los ensayos son los que se recogen en el Cuadro 5.

\section{Cuadro 5}

\begin{tabular}{|c|c|c|c|c|c|}
\hline $\begin{array}{c}\text { Viga } \\
\text { n. }\end{array}$ & $\begin{array}{c}\text { Espesor } \\
\text { capa de } \\
\text { adhesivo } \\
\text { mm }\end{array}$ & $\begin{array}{c}\text { Momento } \\
\text { de } \\
\text { servicio } \\
\mathbf{m K N}\end{array}$ & $\begin{array}{c}\text { Momento } \\
\text { último } \\
\mathbf{M}_{\mathbf{u}} \mathbf{m K N}\end{array}$ & $\begin{array}{c}\text { Momento } \\
\text { de agota- } \\
\text { miento } \\
\mathbf{m K N}\end{array}$ & $\begin{array}{c}\text { Media } \\
\mathbf{m K N}\end{array}$ \\
\hline 21 & 0,7 & 14,8 & 29,7 & 31,1 & 31,5 \\
29 & & & & 32,0 & \\
22 & 1,0 & 14,8 & 29,7 & 32,3 & 32,0 \\
23 & & & & 31,8 & \\
13 & 2,0 & 14,8 & 29,7 & 31,4 & 31,2 \\
14 & & & & 31,0 & \\
15 & 3,0 & 14,8 & 29,9 & 32,4 & 32,3 \\
16 & & & & 32,2 & \\
17 & 5,0 & 15,0 & 30,0 & 32,0 & 31,9 \\
18 & & & & 31,8 & \\
\hline
\end{tabular}




\subsection{Influencia del espesor de la banda de refuerzo}

El espesor de la banda de acero influye en el refuerzo a igualdad de condiciones de resistencia del hormigón, longitud de anclaje, tipo y espesor de adhesivo epoxi utilizado y preparación de superficies. Para una viga dada existe un límite, en cuanto a sección de acero de refuerzo a emplear, debido a que lo ideal sería que se produjese el agotamiento simultáneamente, por plastificación del hormigón comprimido, por rotura del acero en las barras y banda y por agotamiento a cortante del hormigón en los extremos de la misma. Existe, por consiguiente, para una longitud de banda dada, un espesor de la misma que no debe ser superado debido a que si esto ocurre, puede darse el caso de que la tensión de la banda sea muy inferior a su límite elástico cuando falle el refuerzo por cortante del hormigón en los extremos de ella.

Para estudiar la influencia del espesor de la banda de acero en el refuerzo se han empleado vigas tipo en las que las bandas tenían longitudes y anchos de $2,45 \times 0,10 \mathrm{~m}$, pero en las que los espesores se habían supuesto de 3,5 y $8 \mathrm{~mm}$. Los resultados de los ensayos a flexión se indican en el Cuadro 6, en el que además se dan las tensiones en la banda deducidas por deformación de bandas extensométricas para momentos de 26 y $29 \mathrm{mKN}$.

\section{Cuadro 6}

\begin{tabular}{|c|c|r|r|c|}
\hline \multirow{2}{*}{$\begin{array}{c}\text { Viga } \\
\mathbf{n .}^{0}\end{array}$} & \multirow{2}{*}{$\begin{array}{c}\text { Sección de } \\
\text { la banda } \\
\mathbf{c m}^{2}\end{array}$} & \multicolumn{2}{|c|}{$\begin{array}{c}\text { Tensión de tracción en } \\
\text { en } \mathbf{M N} / \mathbf{m}^{2} \text {, para }\end{array}$} & $\begin{array}{c}\text { Momento de } \\
\text { agotamiento } \\
\text { mKN }\end{array}$ \\
\cline { 3 - 5 } & $\mathbf{2 6} \mathbf{~} \mathbf{~ K N}$ & $\mathbf{2 9} \mathbf{~} \mathbf{m K N}$ & \\
\hline 21 & 3,0 & $>316$ & $>316$ & 31,1 \\
29 & & $>316$ & $>316$ & 32,0 \\
27 & 5,0 & 201 & 212 & 34,2 \\
28 & & 193 & 204 & 33,7 \\
30 & \multirow{2}{*}{8,0} & 119 & 128 & 33,8 \\
31 & & 122 & 135 & 34,0 \\
\hline
\end{tabular}

En las vigas 21 y 29 el agotamiento se produjo por plastificación del hormigón después de superar el acero de la banda su límite elástico al igual que ocurrió en las barras de armado; en el resto de las vigas ensayadas el fallo se produjo por agotamiento a cortante del hormigón en el extremo de las bandas de refuerzo. Esto justifica la técnica usualmente utilizada de emplear anclajes de palastro en $\mathrm{L}$ adosados con epoxi en los extremos de las bandas o bien pernos introducidos en el hormigón.

\subsection{Comportamiento frente a fatiga de los refuerzos}

Estos ensayos han tenido por finalidad determinar el comportamiento frente a acciones dinámicas de los refuerzos así como el de los distintos tipos de adhesivos empleados en ellos. Igualmente, se ha comprobado cómo se comportan las bandas de acero que no son enterizas y disponen de soldaduras, de soldaduras con cubrejuntas del mismo espesor de la banda y unidos a ellas mediante la misma formulación epoxi.

Los ensayos se han llevado a efecto sobre vigas reforzadas tipo en las que se han empleado bandas de refuerzo de $2,45 \times 0,10 \times 0,003 \mathrm{~m}$ : unas de una sola pieza y otras formadas por dos bandas soldadas eléctricamente a tope, así como otras que disponían de un cubrejunta de $0,30 \times 0,10 \times 0,003 \mathrm{~m}$ pegada con el mismo adhesivo epoxi del encolado.

Las vigas se han sometido a dos cargas variables situadas a un tercio de la luz que daban 500 ciclos por minuto con momentos comprendidos entre el 50 y $75 \%$ del último de cálculo, así como otro escalón comprendido entre el 60 y $80 \%$ si se superaban $2,10^{6}$ ciclos con el primero.

En el Cuadro 7 se dan resumidos los valores de los ciclos soportados por las vigas hasta la rotura.

En el citado cuadro puede apreciarse cómo las vigas reforzadas con bandas soldadas sin cubrejuntas, rompían en el primer escalón de carga produciéndose la rotura por fallo de la soldadura a fatiga (Figs. 7 y 8 ).

Todas las vigas rompieron por despegue de la banda en sus extremos para los ciclos que se indican, menos la viga reforzada con el Adhesivo A y con banda enteriza que soportó los dos escalones de carga sin que se produjera su agotamiento.

\section{Cuadro 7}

\begin{tabular}{|c|c|c|c|c|c|}
\hline $\begin{array}{l}\text { Viga } \\
\text { n. }\end{array}$ & $\begin{array}{c}\text { Adhesivo } \\
\text { tipo }\end{array}$ & $\begin{array}{c}\text { Banda de } \\
\text { acero }\end{array}$ & $\begin{array}{c}\text { Momento } \\
\text { último } \\
\mathrm{Mu}_{\mathrm{u}} \mathrm{mKN}\end{array}$ & $\begin{array}{c}\text { Ciclos entre } \\
14,8 \text { y } 22,2 \\
\text { mKN }\end{array}$ & $\begin{array}{c}\text { Ciclos entre } \\
17,5 \text { y } 24,7 \\
\text { mKN }\end{array}$ \\
\hline $\begin{array}{l}40 \\
34 \\
54\end{array}$ & $\begin{array}{l}A \\
B \\
C\end{array}$ & Enteriza & 29,7 & $\begin{array}{l}2.10^{6} \\
2.10^{6} \\
2.10^{6}\end{array}$ & $\begin{array}{c}>2.10^{6} \\
3,8.10^{5} \\
4.10^{4}\end{array}$ \\
\hline $\begin{array}{l}52 \\
24\end{array}$ & B & $\begin{array}{c}\text { Soldada } \\
\text { sin } \\
\text { cubre- } \\
\text { junta }\end{array}$ & 29,7 & $\begin{array}{l}9,85.10^{5} \\
7,20.10^{5}\end{array}$ & - \\
\hline 35 & A & $\begin{array}{c}\text { Soldada } \\
\text { con } \\
\text { cubre- } \\
\text { junta }\end{array}$ & 29,7 & $2.10^{6}$ & $1,8.10^{6}$ \\
\hline
\end{tabular}




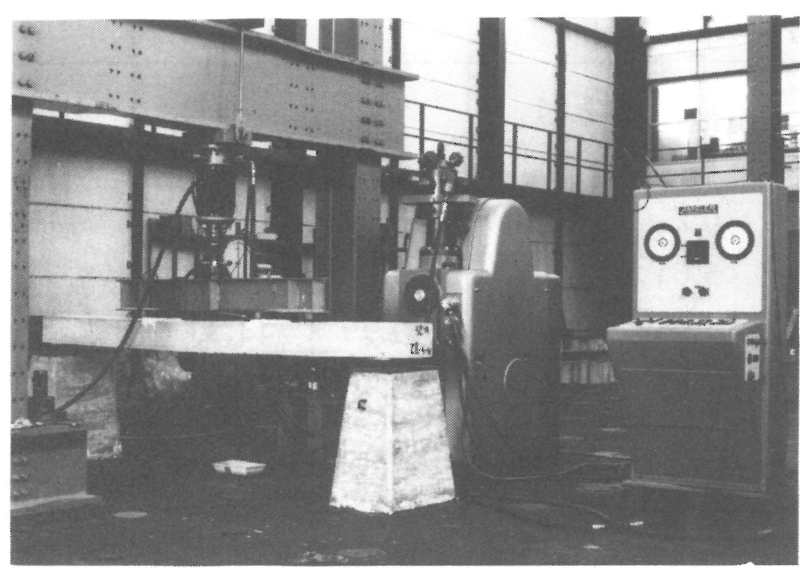

Fig. 7

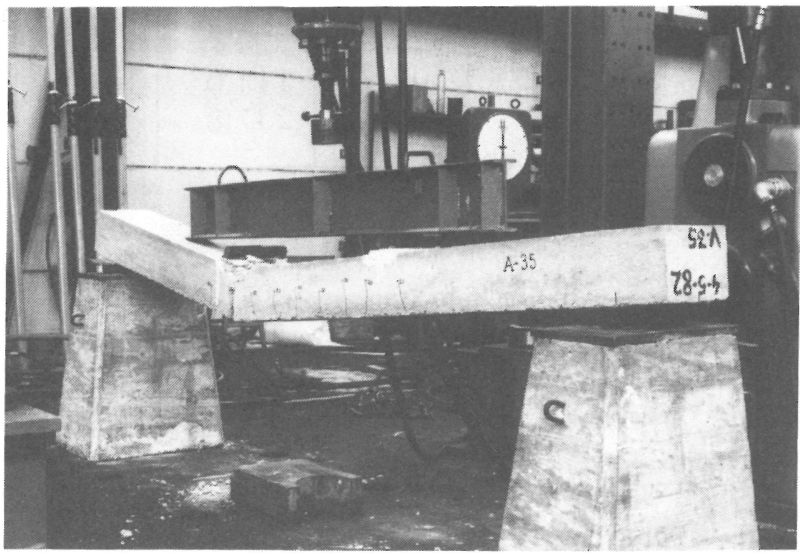

Fig. 8

\subsection{Comportamiento frente a fluencia de los refuerzos}

Los elementos estructurales están proyectados para trabajar de forma continua, es decir, bajo cargas mantenidas; esto hace que sea preciso conocer cómo van a reaccionar frente a este tipo de solicitaciones. Hay que tener en cuenta que un ensayo rápido de una viga reforzada, así como los de fatiga, nos dan una información muy útil, pero no completa, del comportamiento de un refuerzo; es preciso conocer también cómo estos elementos van a reaccionar frente a cargas mantenidas durante años.

Los ensayos de fluencia los hemos realizado sobre vigas reforzadas tipo con bandas de acero de 2,45 $\times 0,10 \times 0,003 \mathrm{~m}$. En ellas se ha tratado de determinar la influencia de los tres tipos de adhesivos empleados, así como el espesor de la capa de los mismos; a este fin, se han estudiado espesores de $0,7 \mathrm{~mm}$ y de $3,0 \mathrm{~mm}$ de adhesivo.

Las vigas a ensayar se han apoyado sobre rodillos separados a 3,00 $\mathrm{m}$ y se han cargado con un contrapeso de hormigón que ejercía su acción sobre dos puntos situados a un tercio de la luz, provocando un momento constante en el centro

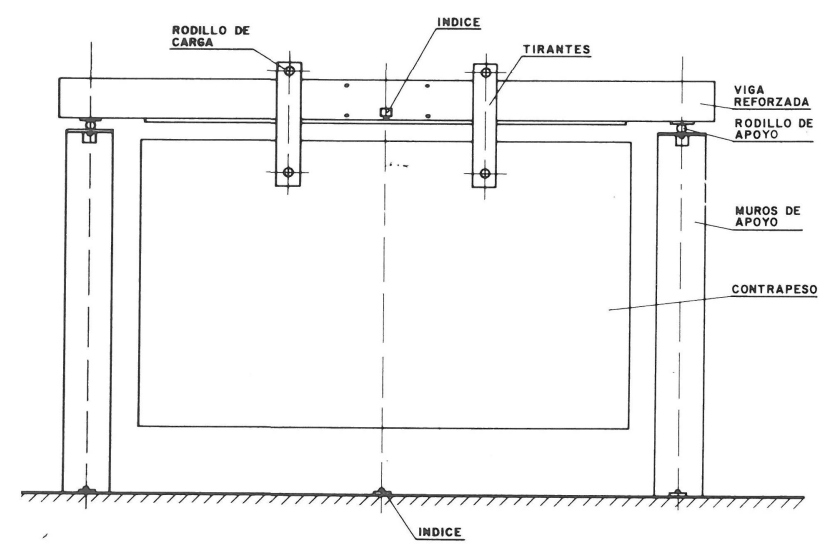

Fig. 9.-Montaje para ensayo de fluencia.

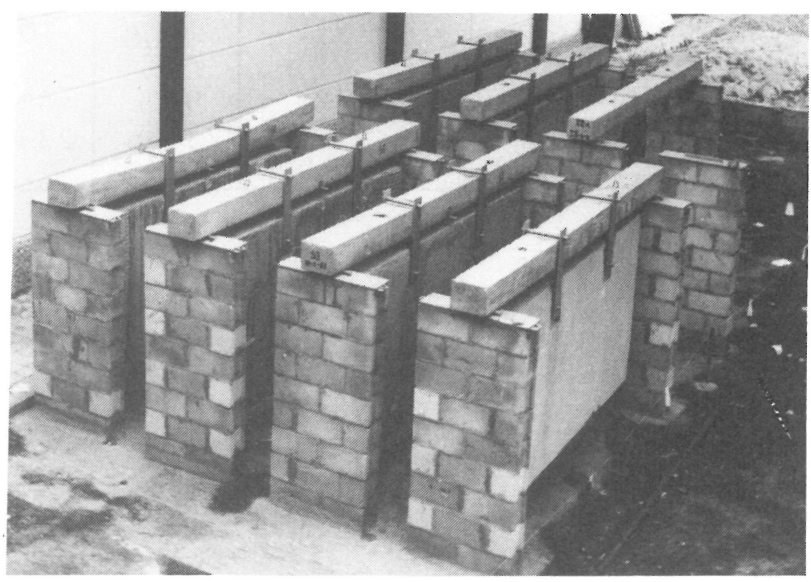

Fig. 10
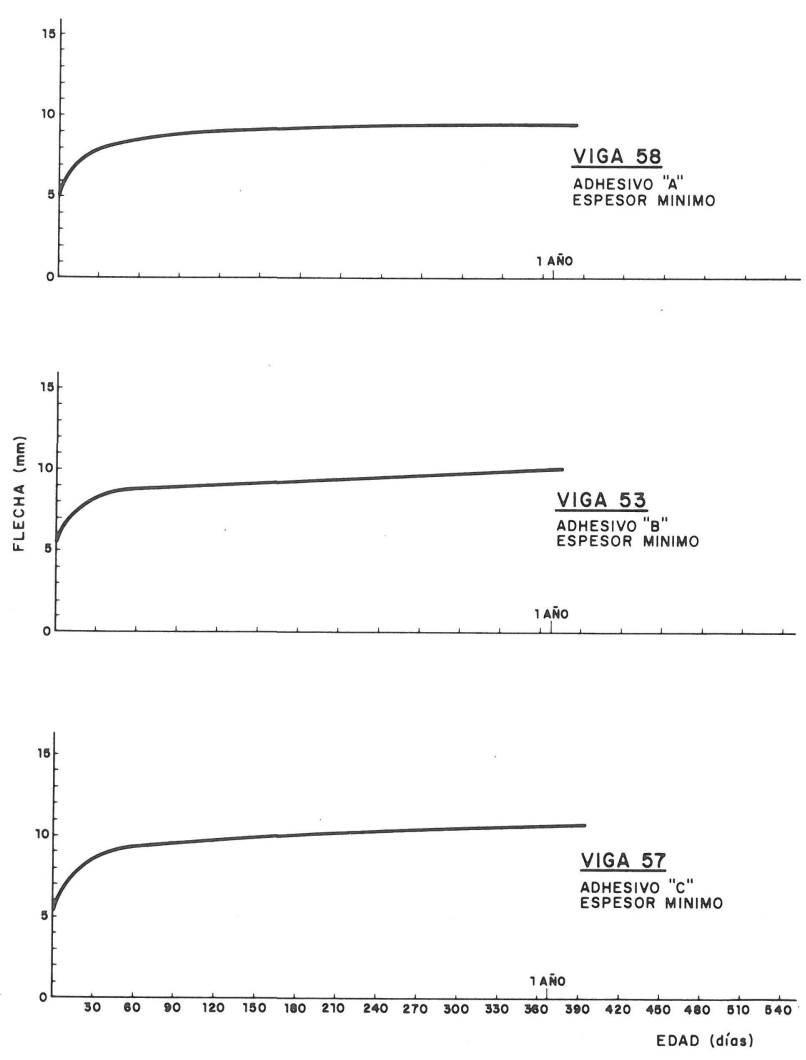

Fig. 11 


\section{Cuadro 8}

\begin{tabular}{|l|c|c|c|c|c|c|c|}
\hline Viga n. $^{\circ}$ & 58 & 53 & 57 & 51 & 56, & 36 & 60 \\
Adhesivo tipo & A & B & C & A & A & B & C \\
Espesor adhesivo (mm) & 0,7 & 0,7 & 0,7 & 2,0 & 3,0 & 3,0 & 3,0 \\
Flecha 1 hora (mm) & 5,51 & 5,60 & 5,61 & 5,52 & 5,50 & 5,62 & 5,60 \\
Flecha al año (mm) & 9,13 & 10,01 & 11,06 & 9,76 & 9,99 & 11,31 & 12,82 \\
Flecha año y medio (mm) & 9,22 & 10,37 & 11,65 & 9,91 & 10,17 & 11,48 & + \\
Incremento de flecha, \% al año & 67,3 & 85,2 & 107,6 & 79,6 & 84,9 & 104,3 & + \\
y medio & & & & & & \\
Incremento de flechas, \% entre & + & - & - & 18,3 & 26,1 & 22,4 & + \\
espesor dado y 0,7 & & & & & \\
Tensión compresión 1 hora y año & 6,72 & 6,60 & 6,80 & 6,54 & 6,60 & 6,95 & 6,60 \\
y medio (MN/m ${ }^{2}$ ) & 9,60 & 10,25 & 9,03 & 9,32 & 9,23 & 11,18 & + \\
Tensión tracción 1 hora y año & 95,5 & 89,5 & 94,4 & 97,2 & 102,5 & 111,0 & 105,2 \\
y medio (MN/m ${ }^{2}$ ) & 230,9 & 233,3 & 236,6 & 242,1 & 226,2 & 237,8 & + \\
\hline
\end{tabular}
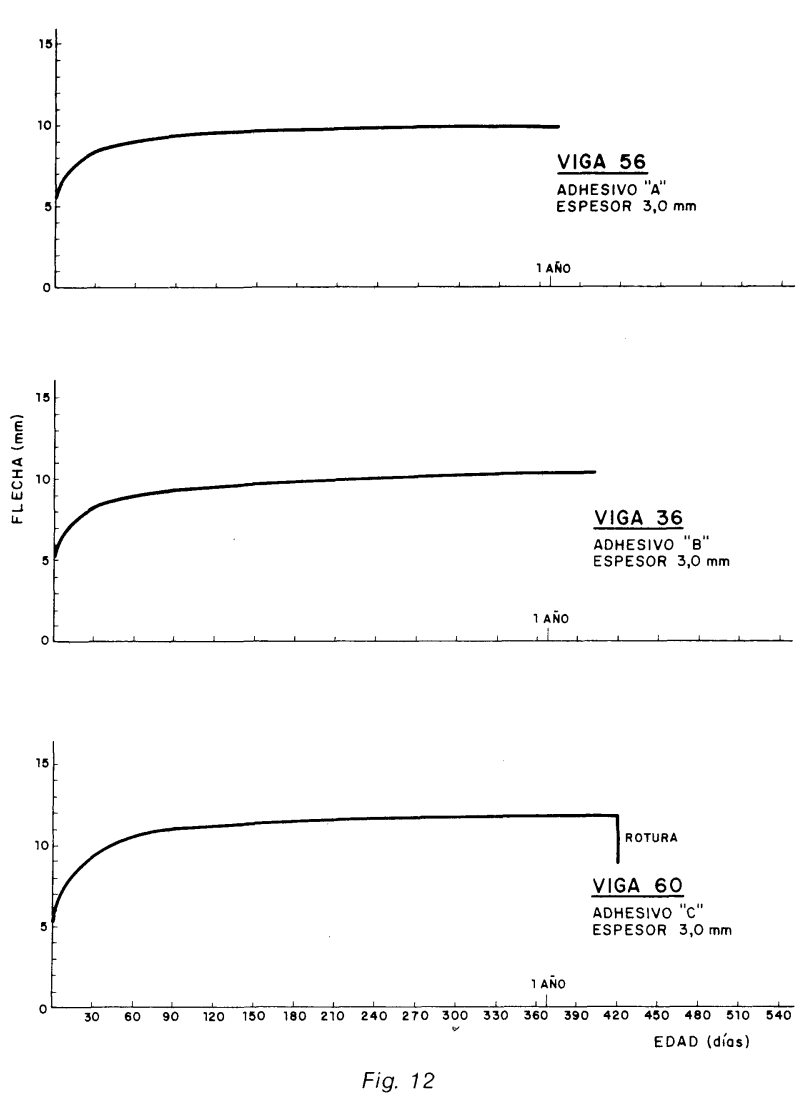

de la viga de 13,9 mKN (Fig. 9). Periódicamente se han medido las flechas en el centro de las vigas, descenso de los apoyos, temperatura ambiente, deformaciones en la zona comprimida y tendida a la altura de las barras de armado, etcétera.

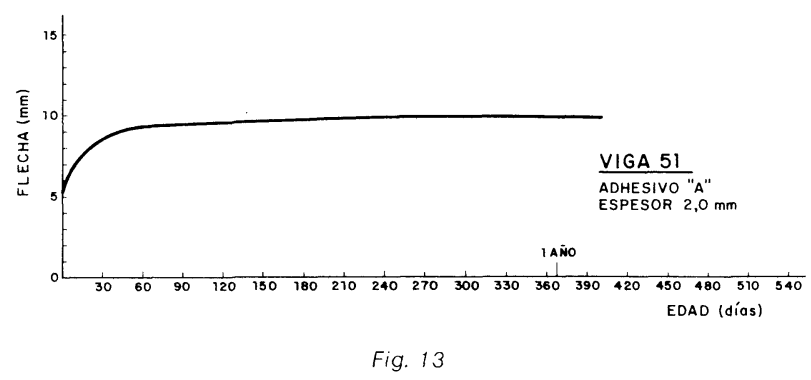

Las vigas están situadas a la intemperie y llevan puestas en carga un año y medio, habiendo soportado temperaturas comprendidas entre $42{ }^{\circ} \mathrm{C} \mathrm{y}-11^{\circ} \mathrm{C}$ (Fig. 10).

Las vigas ensayadas, así como las flechas medidas, tensiones de compresión y tracción en hormigón y barras de armado respectivamente, a la edad de un año y medio, son las que se indican en el Cuadro 8. Los valores de las deformaciones, en función del tiempo, pueden apreciarse en las figuras 11,12 y 13 .

Hay que indicar que la viga $n .{ }^{\circ} 60$ rompio por agotamiento del adhesivo a fluencia a la edad de 420 días (Fig. 14). 


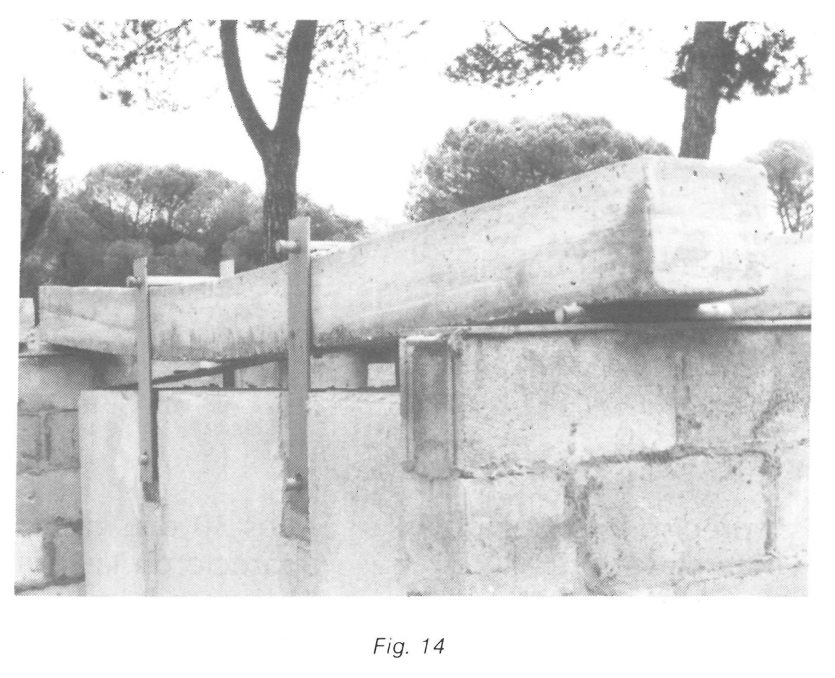

\section{CONCLUSIONES}

1. ${ }^{a}$ El refuerzo de vigas y forjados en estructuras de edificios de viviendas e industriales, mediante el encolado con un adhesivo epoxi de una banda de acero de refuerzo a las superficies del hormigón, es un sistema rápido, fácil, limpio de ejecución y de reducido costo, pero que exige muchos cuidados en cuanto a su proyecto y ejecución.

2. ${ }^{\text {a }}$ Este tipo de refuerzos no debe emplearse con hormigones de resistencia característica inferior a $17,5 \mathrm{MN} / \mathrm{m}^{2}\left(175 \mathrm{kp} / \mathrm{cm}^{2}\right)$. En el Cuadro 2 puede apreciarse el descenso del momento de rotura al disminuir el valor de la resistencia del hormigón. Las roturas en vigas reforzadas en las que el hormigón tiene resistencias bajas suele producirse por fallo a cortante del hormigón en los extremos de la banda de refuerzo.

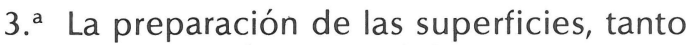
del hormigón como del acero, por los diferentes sistemas ensayados, han demostrado no tener una gran influencia, si bien es verdad que tanto en el escarificado, como en el esmerilado y aplicación del chorro de arena se ha puesto todo el cuidado exigible a un trabajo de laboratorio. Con respecto al chorreado con arena de la banda de acero y posterior pintado con epoxi, que es una técnica muy empleada cuando la preparación se hace en taller, se ha podido apreciar un descenso en la resistencia de rotura que se indica en el Cuadro 3.

El aspecto de las bandas arrancadas después del ensayo puede apreciarse en la figura 15.

4. ${ }^{\text {a }}$ Aunque los tres tipos de adhesivos epoxi eran recomendados por casas conocidas internacionalmente, para este tipo de trabajos, se observa en el Cuadro 4 que el adhesivo «C» tiene ligeramente peor comportamiento que los otros dos. La gran cantidad de carga silícea que poseía y que se apreciaba en su uso, nos hacía sospechar su peor comportamiento que sería puesto en evidencia en los ensayos de fatiga y fluencia.

5. ${ }^{\text {a }}$ El espesor de la capa de adhesivo utilizado en el refuerzo no ha tenido una influencia muy apreciable en los ensayos estáticos realizados según puede apreciarse en el Cuadro 5; sin embargo, sí lo tendría en los ensayos bajo carga mantenida o de fluencia, lo que nos lleva a la conclusión de que debe emplearse el menor espesor posible de adhesivo.

6. ${ }^{\text {a }}$ El espesor de la banda de refuerzo tiene una gran influencia en la eficacia del mismo.

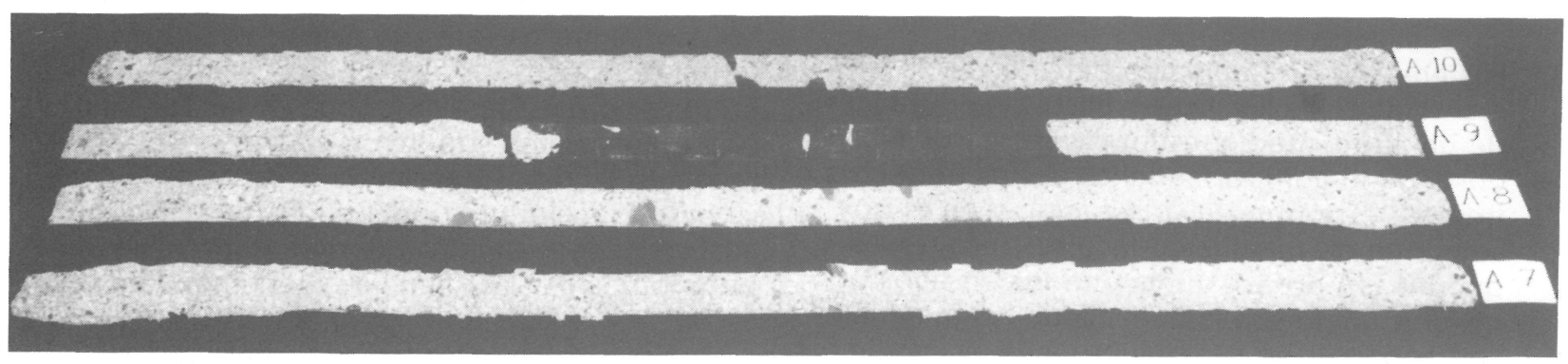


Generalmente, cuando es preciso introducir en el refuerzo un porcentaje elevado de armadura se observa que existe una limitación impuesta por el ancho de la viga a reforzar, lo que obliga a aumentar el espesor de la misma; este aumento de espesor hace que el elemento estructural alcance el agotamiento para tensiones de trabajo de la banda de refuerzo muy reducidas. Los ensayos así lo han manifestado, como puede verse en el Cuadro 6 , en el que se observa cómo al aumentar la sección de acero, con el mismo ancho de banda, no aumenta en igual proporción el momento de agotamiento y cómo las tensiones de trabajo de las bandas, en el momento de producirse el fallo, descienden al aumentar el espesor de la banda. Por consiguiente, no parece conveniente superar los $4,0 \mathrm{~mm}$ de espesor de banda.

7. ' Los ensayos de fatiga han permitido observar, de forma más completa que los estáticos, el comportamiento de los diferentes adhesivos, y así podemos ver en el Cuadro 7 cómo la mayor eficacia en el refuerzo la ha presentado el adhesivo "A» seguido del "B» y la menor la ha presentado el adhesivo «C».

Se ha podido observar también que las bandas unidas por soldadura eléctrica presentan un comportamiento malo; todas rompieron por fallo de la soldadura a fatiga. Si sobre la zona soldada se coloca una platabanda o cubrejunta del mismo espesor de la banda y unido por el mismo adhesivo epoxi, el comportamiento es muy eficaz.

8. ${ }^{\text {a }}$ Los ensayos de fluencia o bajo cargas mantenidas de las vigas y hasta una duración de un año y medio, están permitiendo observar el comportamiento de los refuerzos frente a este tipo de solicitación y en el que se ponen de manifiesto factores más reales que los tenidos en cuenta en los ensayos rápidos, como son la fluencia de los materiales componentes en su conjunto y especialmente la del adhesivo epoxi.
A los 30 días de haber aplicado la carga aparecieron las primeras microfisuras y a los 18 meses la amplitud de éstas, en el tercio central de las vigas, oscilaba entre $0,05 \mathrm{y}$ $0,2 \mathrm{~mm}$, según el adhesivo empleado y el espesor de la capa de éste.

En el Cuadro 8 se observa como el comportamiento fue totalmente normal, salvo para la viga n." 60 , en la que se había empleado el adhesivo «C» en espesor de $3,0 \mathrm{~mm}$.

Cualquiera de los tres adhesivos utilizados con espesores mínimos, y hasta la edad de un año y medio, presentan un comportamiento bueno, aunque como puede apreciarse en el cuadro el valor porcentual de las flechas crece al aumentar el espesor de la capa de adhesivo y con mayor intensidad en el adhesivo «C».

A la vista de lo anterior, se concluye que la fluencia en las vigas reforzadas está influenciada por las características del adhesivo epoxi empleado, así como por el espesor de la capa de éste.

\section{RECOMENDACIONES}

Dado que este trabajo ha venido a completar a los realizados en centros de investigación de otros países y en su parte esencial los resultados obtenidos por nosotros coinciden con los de ellos, las recomendaciones dadas en países tales como Francia, Suiza y Alemania, son aprovechables en nuestro país; no obstante, en nuestro trabajo se ha pretendido estudiar otros parámetros que son típicos de nuestra forma de construir y del empleo de este sistema en nuestro país. Apoyándonos en los resultados obtenidos en nuestra investigación y en las recomendaciones que ha dado el Grupo XII del Comité Euro-International du Beton (CEB), al cual pertenece el autor de este trabajo, nos permitimos dar las siguientes recomendaciones:

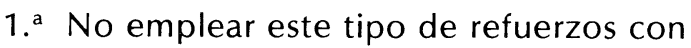
hormigones de baja resistencia. Con este 
sistema no se puede «mejorar» al hormigón y sí sólo introducir una cuantía adicional y limitada de acero en la zona de la sección traccionada.

2. ${ }^{a}$ No emplear esta técnica para incrementar las secciones de acero comprimidas, pues dado el pequeño espesor de las bandas a emplear y el diferente módulo de elasticidad del acero y hormigón, la banda termina por pandear arrancando el adhesivo e incluso al hormigón.

3. ${ }^{\text {a }}$ Antes de realizar la aplicación hay que rectificar la superficie del hormigón, eliminando mediante cincel las cejas e irregularidades producidas por las tablas de los encofrados, hasta conseguir una superficie plana.

4. ${ }^{\text {a }}$ Emplear en la preparación de superficies tanto del hormigón como del acero el chorreado con arena. En casos excepcionales se puede utilizar el escarificado del hormigón.

En cualquier caso, hay que eliminar posteriormente, mediante aspiración, el polvo que se haya podido producir durante las operaciones anteriores.

El acero puede tratarse mediante el ataque rápido con una disolución ácida para eliminar escamas y óxidos, lavando posteriormente con agua y limpiando con tricloroetileno para disolver aceites y grasas.

5. ${ }^{\text {a }}$ Las bandas de acero a utilizar tendrán un espesor que no excederá de $3 \mathrm{~mm}$, según indica el CEB y se ha comprobado en nuestros ensayos; en casos especiales en que se empleen sistemas de anclaje en los extremos de las bandas pueden llegarse hasta espesores de bandas de $10 \mathrm{~mm}$.

6. ${ }^{\text {a }}$ El espesor de la capa de formulación epoxi empleada debe ser el menor posible, no debiendo exceder de $1,5 \mathrm{~mm}$ según indica el CEB y debido, como hemos visto, al efecto negativo que tienen los espesores mayores en las solicitaciones de fluencia y de fatiga.

7. Una vez colocadas en su posición las bandas de refuerzo con el adhesivo aplicado, se ejercerá presión sobre ellas mediante puntales y a través de sopandas, procurando que rebose por los lados el exceso de adhesivo y que finalmente quede el espesor de éste indicado anteriormente.

Los puntales deben ser telescópicos, dotados de rosca y deben ejercer presión durante un tiempo nunca inferior a 24 horas y que será función de la temperatura externa y de la reactividad de la formulación epoxi utilizada.

8. ${ }^{a}$ EI CEB recomienda que cuando una sección se refuerce mediante esta técnica, la capacidad del momento flector adicional no sobrepase la mitad del que tenía la sección antes del refuerzo, es decir, no supere el $50 \%$. Estimamos que la limitación impuesta por el CEB es bastante conservadora y que con la elección de un buen adhesivo y el empleo de un control rígido sobre la ejecución, podría llegarse a valores próximos al $100 \%$.

9. ${ }^{\text {a }}$ El papel del adhesivo epoxi es fundamental en este tipo de refuerzos, especialmente cuando van a estar sometidos a acciones de fatiga.

El adhesivo debe poseer unas características mecánicas adecuadas, reducido coeficiente de dilatación térmica, módulo de rigidez transversal conveniente y además buen poder de mojado y tixotropía idónea para esta aplicación.

Quizá, al ser las formulaciones epoxi mezclas que pueden realizarse con diferentes componentes y de diferentes precios, sea un handicap para ellas debido a que muchos aplicadores desaprensivos, o incluso ignorantes, tienden a conseguir 
formulaciones buscando no razones técnicas, sino simplemente económicas y dando lugar con ello a adhesivos que realmente pegan una banda de acero pero que son incapaces de hacer que ésta trabaje como refuerzo en un elemento de hormigón armado.

Damos a continuación las características mecánicas del adhesivo utilizado por nosotros como patrón, aunque únicamente a título indicativo de una formulación que funciona bien. Desgraciadamente, no podemos indicar valores límites para las diferentes propiedades, debido a que aún no existe una normativa sobre este tema.

Las características del referido adhesivo, son:

Resistencia a compresión a 7 días y $23{ }^{\circ} \mathrm{C}$ medida en probetas de $4 \times 4 \times 16 \mathrm{~cm}$, $82,3 \mathrm{MN} / \mathrm{m}^{2}$.

Resistencia a flexotracción en las mismas condiciones anteriores, 28,9 MN/m².
Resistencia a cizallamiento en encolados acero-hormigón, 6,6 MN/m².

Módulo de elasticidad a $20{ }^{\circ} \mathrm{C}, 16.000 \mathrm{MN} / \mathrm{m}^{2}$.

Módulo de rigidez transversal a $20{ }^{\circ} \mathrm{C}$, $6.600 \mathrm{MN} / \mathrm{m}^{2}$.

Coeficiente de dilatación térmica entre 20 y $50{ }^{\circ} \mathrm{C}, 27 \times 10^{-6} \mathrm{~m} / \mathrm{m}{ }^{\circ} \mathrm{C}$.

Duración de utilización del adhesivo a $20{ }^{\circ} \mathrm{C}$, mínimo 20 minutos, medios sobre $5 \mathrm{~kg}$ de mezcla.

10. ${ }^{a}$ En aquellos casos en que se prevean acciones cíclicas o de fatiga se emplearán bandas de acero enterizas y si por cualquier circunstancia han de llevar soldaduras a tope, se reforzará la zona soldada por un cubrejunta unido a la banda con la misma formulación epoxi.

11. ${ }^{\text {a }}$ La parte reforzada del elemento de hormigón armado debe ser protegida contra los fuertes cambios de temperatura y especialmente frente a los incendios.

\section{BIBLIOGRAFIA}

BRESSON, J.: «Nouvelles recherches et applications concernante la collage dans les structures. Béton plaqué». Annales del Institut Technique du Batiment et Travaux Publics. París, 1971.

MOPU: «Instrucción para el proyecto y la ejecución de obras de hormigón en masa y armado». EH-82. Madrid, 1982.

LADNER, M., y WEDER, CH.: "Concrete structures with bonded external reinforcement». EMPA. Dübendorf. Zurich. Rep. 206 1981.
FERNANDEZ CANOVAS, M.: "Las resinas epoxi en la construcción». Segunda edición. Instituto Eduardo Torroja. Madrid, 1981.

COMITE EURO-INTERNATIONAL DU BETON (CEB): "Assessment of concrete structures and design procedures for upgrading». Redesign.Boletín n. ${ }^{\circ} 162$, octubre, 1983.

FERNANDEZ-CANOVAS, M.: "Patología y Terapéutica del Hormigón Armado». Segunda edición. Editorial Dossat, S. A. Madrid, 1984.

$$
\hat{n} \hat{n} \hat{n}
$$

\section{publicación del i.e.t.c.c.}

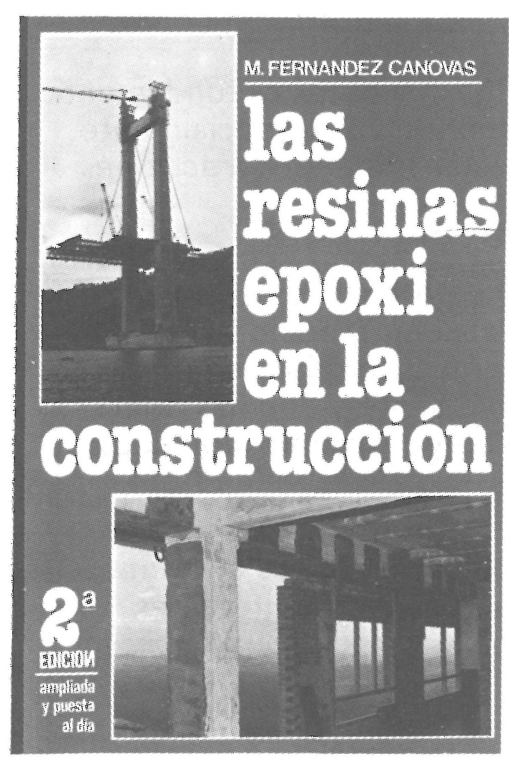

Manuel Fernández Cánovas Dr. Ingeniero de Construcción

Este libro, el primero en lengua castellana sobre resinas epoxi aplicadas a la construcción, está dirigido a arquitectos, ingenieros, constructores y aplicadores. En él, sobre una reducida base teórica imprescindible, se asienta toda una extensa gama de aplicaciones de gran interés.

El autor trabaja desde hace muchos años en el campo de la investigación, especialmente en el estudio de refuerzos y reparaciones estructurales realizados con resinas epoxi.

Con un lenguaje sencillo se tocan todos los problemas que pueden presentarse en la construcción y en los que la solución puede radicar en el correcto empleo de las resinas epoxi.

Se estudian los componentes de las formulaciones epoxi, sus propiedades físicas y quimicas, y aplicaciones, deteniéndose, detalladamente, en las siguientes:

Unión de hormigón fresco a hormigón endurecido. - Unión de hormigones entre si.-Inyecciones de fisuras y grietas. - Unión de acero a hormigón. - Barnices y pinturas. - Las combinaciones brea-epoxi. - Revestimientos de depósitos alimenticios. - Sellado de superficies cerámicas. - Protección de tubos. - Los suelos epoxi en sus diferentes variantes. - Terrazo epoxi. - Reparación de baches. - Reparación de desperfectos en estructuras. - Reparación de carreteras de hormigón. - Juntas elásticas. - Guardacantos de tableros de puentes. - Refuerzos de pilares, vigas, forjados y zapatas, etc. - Consolidación de suelos. - Anclajes. - Protección de aceros en pretensado.

Se termina con unos capitulos dedicados a la limpieza y preparación de las superficies según los materiales a unir; al control del estado superficial de éstos; a las condiciones de temperatura de aplicación; limpieza de los útiles de trabajo; precauciones en el manejo de los sistemas; almacenaje, mezcla y manejo de las formulaciones epoxi $y$ métodos de ensayo de sistemas y aplicaciones epoxidicas.

Un volumen encuadernado en cartoné plastificado con lomo de tela, de $17 \times 24 \mathrm{~cm}$, compuesto de 334 páginas y 158 figuras y fotografías.

Madrid, 1981.

Precios: España, 1.700 ptas.; extranjero, \$ USA 34.00. 